\title{
Emergence et diffusion d'items identitaires et culturels : le cas de la communauté en ligne 9GAG
}

\begin{abstract}
Albin Wagener ${ }^{1}$
Depuis 2008, le site communautaire 9gag rassemble des utilisateurs du monde entier en leur permettant de mettre en ligne des publications (images, vidéos, petites histoires) visibles par tous les internautes, sans se concentrer sur les relations entre utilisateurs. Les publications sont pour la plupart rédigées en anglais, afin de favoriser le développement du site et l'échange d'items culturels.Mais si certaines frontières sont effectivement franchies, c'est au prix d'une seule langue et d'éléments culturels majoritairement anglo-américaines. Ainsi, les publications dans d'autres langues se retrouvent critiquées par les utilisateurs, alors que certains pays ou groupes sont régulièrement la cible de moqueries. Dans cette perspective, on retrouve ici la prévalence des prédiscours (Paveau, 2006) et la reproduction d'items culturels (Enfield \& Levinson, 2006) afin d'assurer une popularité maximale des publications. Notre analyse de cette communauté d'utilisateurs porte sur un corpus de 446 publications 9 gag mises en ligne entre août et septembre 2012. A travers cette étude, nous souhaitons explorer deux questions : 1) est-il possible de rassembler plusieurs univers symboliques sans utiliser les mêmes références communes ou une même langue (Bucholz \& Hall, 2005) ; 2) se pourrait-il que les références anglo-américaines ne soient plus la propriété de leur aire culturelle d'origine, mais qu'elles soient utilisées afin de constituer de nouveaux éléments de communalité (Lemieux, 2009) ? Au cours de cette analyse, nous garderons à l'esprit
\end{abstract}

1 Albin Wagener est Chercheur à l'Université catholique de l'Ouest et Directeur de l'Institut du perfectionnement en langues vivantes.

Recherches en communication, $\mathrm{n}^{\circ} 40$ (2013). 
que la culture est ici utilisée comme excuse pour permettre l'effacement des différences (Dervin, 2011 ; Wagener, 2012) afin de construire une identité imaginée qui pourrait échapper aux troubles du monde en dehors d'internet (Boudon, 2005).

Depuis 2008, le site communautaire 9gag rassemble des utilisateurs du monde entier en leur permettant de mettre en ligne des publications (images, vidéos, petites histoires) visibles par tous les internautes. Contrairement aux réseaux sociaux, 9 gag constitue une communauté qui partage uniquement des publications (ou " posts »), sans se concentrer sur les relations entre utilisateurs. Les publications sont pour la plupart rédigées en anglais, afin de favoriser le développement transnational du site et l'échange de différents items culturels. Malgré cette visée de départ, la réalité du site se révèle être bien plus complexe ; en effet, si certaines frontières sont effectivement franchies, c'est au prix d'une seule langue et d'éléments culturels majoritairement anglo-américains. Ainsi, les publications dans d'autres langues se retrouvent critiquées par les utilisateurs, alors que certains pays ou groupes sont régulièrement la cible de moqueries. Dans cette perspective, on retrouve ici la prévalence des prédiscours (Paveau, 2006) et la reproduction d'items culturels (Enfield \& Levinson, 2006) afin d'assurer une popularité maximale des publications. Notre analyse de cette communauté d'utilisateurs porte sur un corpus de 446 publications 9gag mises en ligne entre août et septembre 2012. A travers cette étude, nous souhaitons explorer deux problématiques principales : 1) est-il possible de rassembler plusieurs univers symboliques sans utiliser les mêmes références communes (Bucholz \& Hall, 2005) ; 2) comment fonctionne cette mise en commun d'éléments sémantiques, sur la base de quels référents et quels mécanismes ? Permettent-ils de constituer de nouveaux éléments de communalité (Lemieux, 2009) et à quel prix ? Au cours de cette analyse, nous garderons à l'esprit que la culture est ici utilisée comme excuse pour permettre l'effacement des différences (Dervin, 2011 ; Wagener, 2012) afin de construire une identité imaginée qui pourrait échapper aux troubles du monde en dehors d'internet (Boudon, 2005).

\section{9gag : une communauté d'échanges en ligne}

Créé en 2008 par un groupe d'internautes, le site internet 9gag se définit, d'après les gestionnaires du site eux-mêmes, comme " the 
funniest yet simplest platform for you to publish or collect funs $»^{1}$. Au fur et à mesure des années, 9gag s'est imposée de façon progressive comme une plateforme incontournable, où les utilisateurs peuvent publier des images, des vidéos ou d'autres formes d'expressions typiques d'internet. Le site communautaire 9gag s'inscrit dans une tradition établie d'autres sites communautaires de type alternatif, desquels naissent les modes d'expression des internautes que l'on pourra retrouver sur divers types de forums ou de sites divers. Ainsi, avec 4chan, Memebase, Rageshare ou encore IraffIruse, 9gag rassemble des internautes actifs, issus de tous pays, classes sociales ou âges, et partageant diverses sources d'intérêt. Contrairement aux réseaux sociaux comme Facebook ou Myspace, $9 \mathrm{gag}$ ne se focalise pas sur les individus ou les relations qui peuvent se nouer entre eux, mais uniquement sur les productions ou « posts ». On retrouve dans ces « posts » des éléments typiques de l'expression de ce type de sites, comme les « memes » ou les « ragecomics ». Les premiers sont une réduction intellectuelle héritée de la théorie des memes (Dennett, 1990, p. 128), soit la reproduction sociale et la transmission culturelle d'idées et de concepts ; les seconds constituent de très courts développements inspirés directement de l'univers de la bande dessinée et adaptés aux contraintes d'internet, en se basant notamment sur l'utilisation de " ragefaces », soit des dessins de visages stéréotypés représentant des émotions précises et aisément compréhensibles par la communauté d'internautes. En d'autres termes, l'univers duquel se réclame 9gag possède ses propres codes d'utilisation, afin de rassembler et de souder une communauté d'utilisateurs toujours plus nombreuse.

Pour ce faire, les concepteurs et utilisateurs de 9gag ont très rapidement généralisé l'utilisation de la langue anglaise, dans une optique transnationale de rassemblement : en produisant des " posts » en langue anglaise, il serait plus aisé de toucher un maximum de personnes de par le monde. Certains utilisateurs tentent d'imposer d'autres langues (espagnole, allemande, française, etc.) mais ceci se solde par des échecs répétés, notamment par un système de filtrage des publications proposées et soumises aux votes des utilisateurs. En effet, le site est subdivisé en trois catégories de publications :

- la première, "What's hot ", rassemble les publications les plus populaires, soit celles qui ont rassemblé le plus de votes des utilisateurs ;

1 « La plateforme la plus amusante et la plus simple pour y publier ou collecter des messages amusants »; notre traduction. Source : http://9gag.com/faq 
- la seconde, «Trending », présente les publications en tête des votes, et qui nécessitent encore quelques efforts de la part des utilisateurs pour se retrouver sur la page « What's hot »;

- la dernière enfin, "Fresh ", présente les publications directement proposées par les utilisateurs, qui les soumettent ainsi directement au vote de la communauté.

En d'autres termes, si ce principe permet de classer les publications par popularité (et souvent d'évacuer des publications à caractère problématique), c'est bien le principe du vote majoritaire qui va permettre ou non l'émergence et/ou la diffusion d'items culturels propres à la communauté d'internautes. Dans cette optique, le système préconisé par 9gag constitue un laboratoire intéressant d'échanges et d'impositions de tendances culturelles propres, avec ses avantages et ses limites.

\section{Prédiscours et mise en scène des identités culturelles}

Si le site internet $9 \mathrm{gag}$ veut briser les frontières entre les internautes pour les rassembler, l'analyse d'un corpus de publications mises en ligne sur le site peut mettre en lumière la complexité nuancée de la réalité. Afin d'étayer notre étude d'un corpus de 446 publications, nous souhaitons mettre en relief trois principes fondamentaux qui constituent notre cadre théorique. Le premier principe concerne la théorie des prédiscours (Paveau, 2006), dans la mesure où nous estimons que les utilisateurs de 9gag ne sont pas vierges de toute influence culturelle, éducative, psychologique ou sociale, et qu'ils peuvent parfois finir par rejouer des formes de discrimination latente, malgré la visée rassembleuse du site. Dans cette optique, la théorie développée par Marie-Anne Paveau met en lumière le rôle joué par les prédiscours, notamment dans la reproduction de catégorisations cognitives, mais également dans la persistance de certains schémas dits culturels (Paveau, 2006, p. 14) :

Les prédiscours sont en effet des opérateurs dans la négociation du partage, de la transmission et de la circulation du sens dans les groupes sociaux. Je les définis comme un ensemble de cadres prédiscursifs collectifs qui ont un rôle instructionnel pour la production et l'interprétation du sens en discours. J'entends par prédiscours des contenus sémantiques (au sens large de culturel, 
idéologique, encyclopédique), c'est-à-dire des savoirs, des croyances et des pratiques.

Appliquer cette définition des prédiscours à la communauté active sur 9gag permet d'expliquer comment la production des publications va véhiculer un cadre sémantique déjà préconstruit ou, pour le moins, dont une partie porte un germe de reproduction sociale ou culturelle, susceptible de déclencher une interprétation du sens compréhensible par un nombre important d'utilisateurs, en fonction des contraintes communicationnelles du site. Dans le cas de 9gag, la persistance du mécanisme des prédiscours est couplée avec l'utilisation de la culture comme modulateur, selon la définition d'Enfield et Levinson. En effet, selon eux, la culture elle-même ne constitue pas l'essence ou la monnaie d'échange des interactions, mais n'en est qu'une coloration circonstanciée (Enfield \& Levinson, 2006, p. 29). Ainsi, les interactions seraient motivées par l'utilisation de prédiscours, eux-mêmes possiblement modulés ou adaptés par des tendances culturelles qui ne modifient pas considérablement des tendances humaines qu'Enfield et Levinson considèrent comme étant universelles - comme le besoin de vivre en groupe ou de s'organiser au sein d'une société, par exemple. Cette définition, que nous utilisons pour la présente étude, se rapproche également de ce que Paul Kay appelait la boîte à outils culturelle, dans la mesure où l'on n'utilise jamais une culture dans sa totalité (et qu'une telle totalité n'existe pas), mais uniquement certains éléments en fonction des situations (Kay, 1999, p. 110).

Pour mieux comprendre les mécanismes qui articulent la circulation des échanges sur la plateforme 9gag, il est également nécessaire d'apporter une définition de l'identité, dans la mesure où celle-ci se trouve ici mise en scène en ligne, dans un cadre défini par un certain nombre de contraintes, afin de favoriser les échanges entre utilisateurs répartis sur toute la surface du globe. Dans cette optique, la théorie de l'identité développée par Burke et Stets, malgré ses limites souvent trop fractionnées (notamment la définition de l'identité comme agent social, parfois déconnecté de l'individu), nous permet malgré tout de délimiter les caractéristiques typiques de la signification identitaire et de son utilité sociale (Burke \& Stets, 2009, p. 3).

An identity is the set of meanings that define who one is when one is an occupant of a particular role in society, a member of a particular group, or claims particular characteristics that identify 
him or her as a unique person. (...) People possess multiple identities because they occupy multiple roles, are members of multiple groups, and claim multiple personal characteristics, yet the meanings of these identities are shared by members of society ${ }^{1}$.

Ainsi, puisque la signification des identités est une donnée partagée par les membres d'une société ou même d'une communauté définie, nous pouvons supposer que les utilisateurs de 9gag auront tendance à attendre ou reproduire la mise en scène de certains mécanismes identitaires, que ce soit sur la base (cognitive et émotionnelle) des prédiscours et de leur utilité sociale, ou bien sur la base de la culture comme coloration spécifique et comme outil de partage - un outil qui n'échappe jamais, comme nous pourrons le constater, aux enjeux de pouvoir (Angouri \& Glynos, 2009).

\section{Questions et méthodologie}

A travers la présentation de ce site communautaire et le cadre théorique que nous avons délimité, nous souhaitons poser deux questions distinctes qui nous semblent fondamentales :

- Est-il possible de rassembler différents univers symboliques sans pour autant utiliser une seule langue ou un seul ensemble de références communes? 9gag ne semble en effet pas réussir à échapper à cette contrainte forte ;

- Les références anglo-américaines largement échangées sur 9gag sont-elles encore des propriétés de leurs aires culturelles d'origine, ou bien sont-elles devenues une monnaie d'échange nécessaire à la mondialisation, progressivement vidées de leur signification initiale, et sources d'une nouvelle source sémantique transnationale?

1 «Une identité est un ensemble sémantique qui définit un individu lorsque celui-ci occupe un rôle particulier dans la société, est membre d'un groupe en particulier, ou se réclame de caractéristiques spécifiques qui l'identifie comme une personne unique. (...) Les individus possèdent plusieurs identités, dans la mesure où ils occupent des plusieurs rôles, sont membres de plusieurs groupes et se réclament de plusieurs caractéristiques personnelles ; cependant, les significations de ces identités sont partagées par les membres de la société »; notre traduction. 
Pour tenter de répondre à ces deux questions, nous avons décidé d'analyser un corpus de publications mises en ligne sur le site internet. Nous avons choisi uniquement les publications qui apparaissent sur la page «What's hot », en ce qu'elles ont remporté la majorité des votes des utilisateurs de $9 \mathrm{gag}$ et témoignent ainsi d'une popularité de certains éléments récurrents. Nous avons pu ainsi rassembler et analyser un corpus de 446 publications, toutes mises en ligne entre le 5 août 2012 et le 3 septembre 2012. Ainsi, en un mois environ, nous avons choisi d'effectuer quatre prélèvement distincts et aléatoires (5 août, 11 août, 23 août et 3 septembre 2012), effectués sur l'ensemble des journées précisées (sur 24 heures précisément, de la première à la dernière publication de la journée sur la page « What's hot ? »), que nous rassemblons ici.

Afin d'analyser ces échantillons et d'en étudier la complexité, nous choisissons d'utiliser une méthode d'analyse qui combine trois principes simples et néanmoins fondamentaux, à savoir :

- $\quad$ le traitement superficiel de Maillat \& Oswald, qui indique que les lecteurs et/ou récepteurs d'un discours utilisent la plupart du temps des raccourcis cognitifs, afin d'économiser de l'énergie et de faciliter la cohérence pour la compréhension d'un message spécifique (Maillat \& Oswald, 2009). Dans cette perspective, les lecteurs/récepteurs utilisent des concepts ad hoc lors de l'interprétation d'un message, dans un esprit général de confort cognitif;

- la sémantique des possibles argumentatifs de Galatanu (ou modèle SPA), qui explique la manière dont les lecteurs utilisent leurs représentations sémantiques dans un nuage topique donné, afin de recréer un environnement contextuel cohérent (Galatanu, 2003) : cette méthode s’inspire directement de l'analyse linguistique et discursive, et présente un lien pertinent avec l'hypothèse du traitement superficiel ;

- $\quad$ le modèle STA de Cap (spatial/temporel/axiologique), utilisé notamment en analyse des discours politiques et basé sur la pragmatique, fait état quant à lui des effets de l'impression de proximité spatiale, temporelle et axiologique sur la compréhension d'un discours par un lecteur/récepteur et l'attribution de certaines caractéristiques sémantiques (Cap, 2010). 
L'utilisation de ces trois principes combinés nous permet de comprendre que les utilisateurs de 9gag (qu'ils soient producteurs ou récepteurs des publications en ligne) vont tenter de recréer un environnement sémantique cohérent et peut-être même déjà connu, sur la base des trois mécanismes suivants postulés par les principes susnommés :

- le traitement superficiel des informations est favorisé par l'utilisation de référents immédiatement identifiables par les internautes utilisateurs de 9gag, notamment la mention de séries télévisées anglo-saxonnes ;

- les nuages topiques ainsi générés permettront à la communauté 9gag de relier des éléments sémantiques propres aux utilisateurs, et de produire un mécanisme d'auto-référence systématique qui sera partagé et reproduit par bon nombre de publications populaires ;

- ces éléments référentiels vont ainsi mettre en relief une proximité axiologique forte des référents utilisés pour véhiculer des messages, afin de rassembler une communauté disséminée sur l'ensemble du globe, malgré l'éloignement géographique, au profit d'une immédiateté temporelle.

De surcroit, les prédiscours comme outils d'aide à la construction et au décodage d'un environnement cohérent permettent également de comprendre les liens entre ces trois mécanismes, dans la mesure où cette reconstruction pourra également obéir à une proximité mesurable de certains éléments sociaux, culturels ou identitaires, qui sont immédiatement accessibles (et donc utilisables et décodables) par le plus grand nombre d'utilisateurs, et donc susceptibles de recueillir un nombre maximal de votes. Ce processus intervient à deux niveaux :

- $\quad$ soit pour donner du sens aux publications mises en ligne (dans une optique de compréhension du message et d'assimilation des codes du site);

- $\quad$ soit pour reproduire un sens et l'imposer comme une entité relativement bien partagée (notamment en fonction de l'importance des votes).

- La complexité nuancée de ce processus fait de 9gag un véritable laboratoire, en ce qu'il permet aux utilisateurs de faire évoluer les codes et les significations qu'ils produisent 
et utilisent, tout en favorisant l'émergence et le déclin d'items culturels.

\section{Extraction statistique des données du corpus}

Afin de pouvoir étudier de manière précise la complexité de ce corpus de 446 publications publiées sur la page « What's hot? » du site communautaire 9gag, nous avons tout d'abord choisi de les catégoriser par thématiques. En fonction des thématiques étudiées, il est ensuite possible de délimiter les significations qui y étaient majoritairement associées et d'en analyser les possibles impacts sur la compréhension des occurrences sémiotiques. Nous avons isolé 21 catégories distinctes qui témoignent de la grande variété des sujets abordés, et avons observé leur répartition en fonction des quatre journées de prélèvement, ce qui permet de proposer le tableau suivant :

\begin{tabular}{|c|c|c|c|c|c|c|c|}
\hline & $05 / 08 / 2012$ & $11 / 08 / 2012$ & 23/08/2012 & $03 / 09 / 2012$ & en nombre & $\underline{e n \%}$ & $\underline{\text { moyenne }}$ \\
\hline 1 - Animaux & 4 & 11 & 15 & 8 & 38 & $8,52 \%$ & 9,5 \\
\hline 2 - Autoréférences à 9gag & 1 & 0 & 0 & 1 & 2 & $0,45 \%$ & 0,5 \\
\hline 3 - Connotations sexuelles & 7 & 7 & 5 & 2 & 21 & $4,71 \%$ & 5,25 \\
\hline 4 - Créativité et arts & 1 & 1 & 9 & 4 & 15 & $3,36 \%$ & 3,75 \\
\hline 5 - Comics, Memes, Ragefaces & 1 & 6 & 6 & 4 & 17 & $3,81 \%$ & 4,25 \\
\hline 6-Nature & 0 & 0 & 1 & 5 & 6 & $1,35 \%$ & 1,5 \\
\hline 7 - Nourriture & 5 & 3 & 7 & 2 & 17 & $3,81 \%$ & 4,25 \\
\hline 8 - Cultures et pays étrangers & 5 & 8 & 3 & 2 & 18 & $4,04 \%$ & 4,5 \\
\hline 9 - Films et séries & 0 & 5 & 14 & 7 & 26 & $5,83 \%$ & 6,5 \\
\hline 10 - Internet, techniques, science & 10 & 8 & 12 & 14 & 44 & $\mathbf{9 , 8 7 \%}$ & 11 \\
\hline 11 - Jeux vidéo & 3 & 4 & 5 & 4 & 16 & $3,59 \%$ & 4 \\
\hline 12 - Mangas et dessins animés & 2 & 7 & 1 & 4 & 14 & $3,14 \%$ & 3,5 \\
\hline 13 - Musique & 0 & 0 & 4 & 2 & 6 & $1,35 \%$ & 1,5 \\
\hline 14 - Sport & 8 & 10 & 3 & 2 & 23 & $5,16 \%$ & 5,75 \\
\hline 15 - Relations hommes/femmes & 14 & 8 & 11 & 5 & 38 & $8,52 \%$ & 9,5 \\
\hline $\begin{array}{l}16 \text { - Relations humaines, amis, } \\
\text { famille }\end{array}$ & 3 & 11 & 13 & 7 & 34 & $7,62 \%$ & 8,5 \\
\hline 17 - Religion & 1 & 2 & 1 & 0 & 4 & $0,90 \%$ & 1 \\
\hline 18 - Société, histoire et politique & 10 & 2 & 10 & 7 & 29 & $6,50 \%$ & 7,25 \\
\hline 19 - Télé et médias & 0 & 0 & 4 & 1 & 5 & $1,12 \%$ & 1,25 \\
\hline 20 - Travail et études & 2 & 3 & 8 & 4 & 17 & $3,81 \%$ & 4,25 \\
\hline 21 - Vie quotidienne & 11 & 11 & 21 & 13 & 56 & $12,56 \%$ & 14 \\
\hline \multirow[b]{2}{*}{ TOTAL POSTS PAR JOUR (PPJ) } & 88 & 107 & 153 & 98 & 446 & $100,00 \%$ & 111,5 \\
\hline & $19,73 \%$ & $23,99 \%$ & $34,30 \%$ & $21,97 \%$ & $100,00 \%$ & & \\
\hline
\end{tabular}

Tableau 1. Répartition des publications ou «posts » par jour et par catégorie. 
Si la variété des thématiques abordées est clairement visible, certaines semblent plus sollicitées que d'autres, notamment la vie quotidienne, les relations humaines, les animaux ou encore les références à internet et aux techniques. D'autres, comme la religion, les médias ou les autoréférences au site 9 gag sont quasi absentes du corpus. Il est de surcroît intéressant de noter qu'en fonction des utilisateurs, de leurs productions et de leurs votes, la quantité de publications référencées sur la page "What's hot ? » oscille sensiblement en fonction des dates. Il est également utile de préciser que les publications liées au sport étaient assez élevées, ce notamment en raison de l'actualité du mois d'août 2012, puisque la ville de Londres accueillait des Jeux Olympiques d'été fortement médiatisés.

Après avoir répartis les différentes publications par thématiques et de les avoir regroupées sans distinction de date, nous avons pu constater un phénomène intéressant et pour le moins étonnant ; si en effet, les utilisateurs de 9gag tentent de maintenir une communauté qui partage des références qui traversent les frontières nationales (et éventuellement sociales), ce rassemblement semble s'effectuer en grande partie sur la reproduction et la diffusion de stéréotypes concernant certains groupes sociaux. En d'autres termes, le rapprochement entre les différents utilisateurs se ferait aux dépens de certaines catégories sociales ou sociétales, qui se retrouvent ici la cible de moqueries qui, derrière le masque apparent de l'humour partagé, dissimulent mal l'utilisation d'internet comme outil et agent de discrimination.

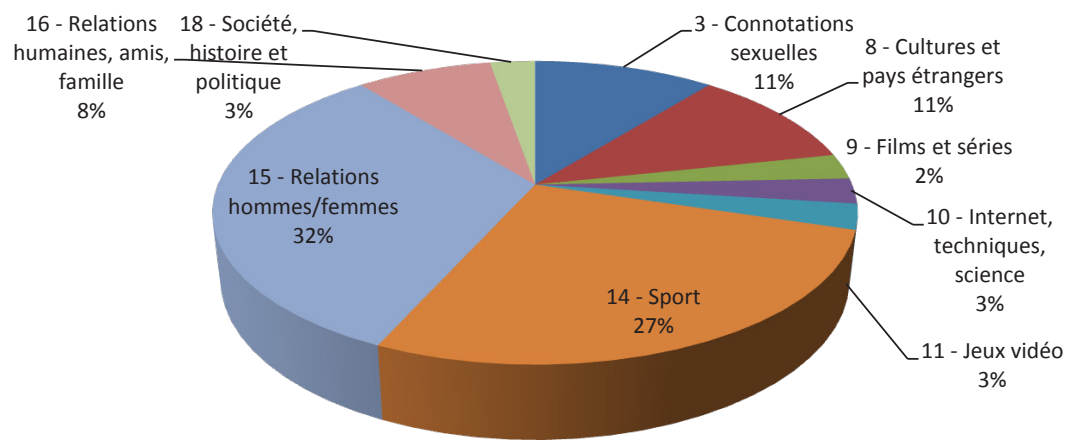

Image 1. Occurrences de stéréotypisations par thématique 
Les relations entre hommes et femmes se trouvent ainsi fortement porteuses de discrimination, notamment envers les femmes ; le même phénomène se produit au niveau du sport, les Jeux Olympiques de Londres d'août 2012 devenant l'occasion de mettre en lumière des moqueries répétées au sujet de sports typiquement féminins (comme la natation synchronisée) ou de publier des photos de jeunes athlètes, où la sportive performante devient un simple objet sexuel à contempler. En approfondissant 1'analyse de ces discriminations, nous constatons qu'elles concernent cinq thématiques principales.

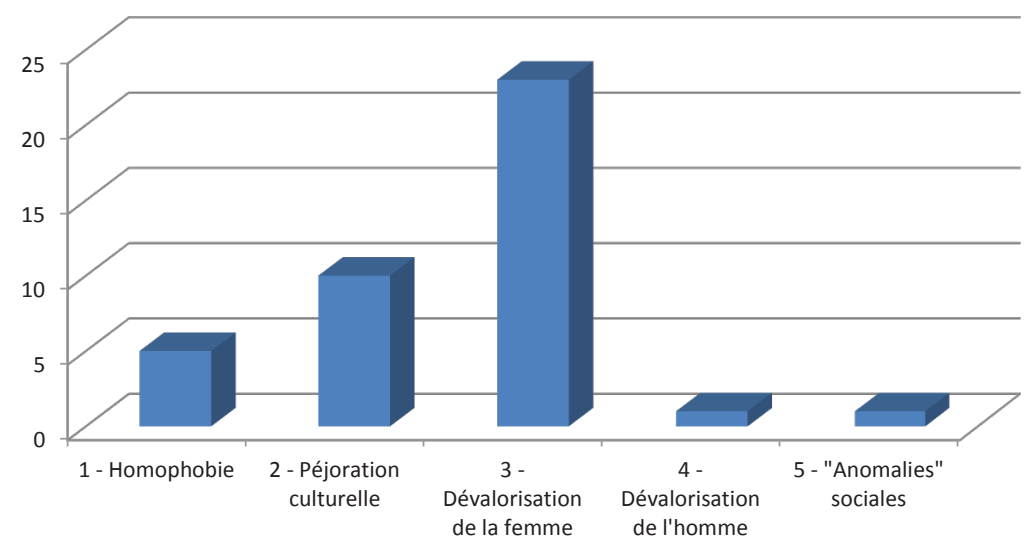

Image 2. Types de stéréotypisations

Au total, nous avons repéré 40 publications clairement identifiables comme discriminantes, ce qui fait un total de $11,15 \%$ de publications dirigées contre certains groupes. Ainsi, pour les utilisateurs de 9gag, 1 publication sur 10 fait l'objet de discriminations aisément identifiables, ce qui est loin d'en faire un site rassembleur. Pour illustrer notre propos, nous souhaitons simplement soumettre au lecteur les trois exemples suivants, qui témoignent de ces phénomènes discriminatoires dans les publications : 


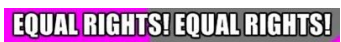
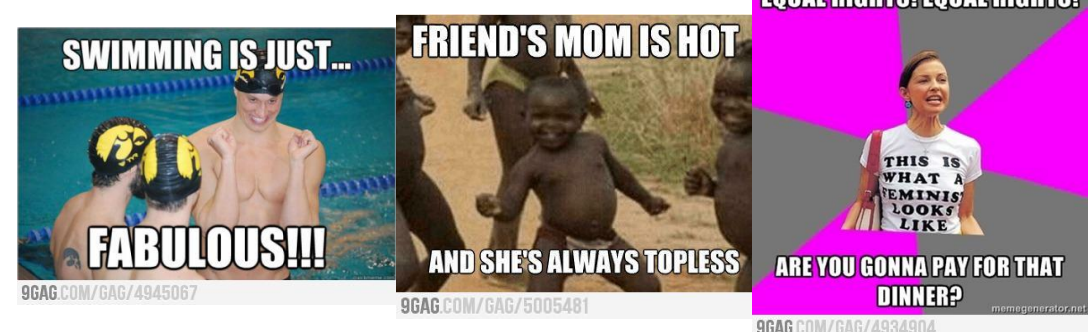

Illustration 1. trois exemples de publications discriminantes.

Ces trois illustrations permettent d'exemplifier ce que le graphique 2 présente, à savoir la persistance d'un «trio de tête » en matière de discrimination : dévalorisation de la femme, péjoration culturelle, et une sorte d'" homophobie ordinaire » qui invite, par le biais de la moquerie, à se conformer à un stéréotype de mâle hétérosexuel. Ces discriminations ne sont pourtant pas le reflet de la communauté 9gag, qui regroupe elle-même des gens de tous horizons et de tous groupes ; si le site internet est loin d'être le terrain de jeu de mâles hétérosexuels blancs, il semble pourtant en arborer tous les attributs.

Cette première déduction se trouve renforcée par une dernière analyse des publications de 9gag, qui permet de mesurer le niveau de mixité culturelle encouragée par la communauté d'utilisateurs. Ici encore, nous nous retrouvons face à un constat objectif intéressant :

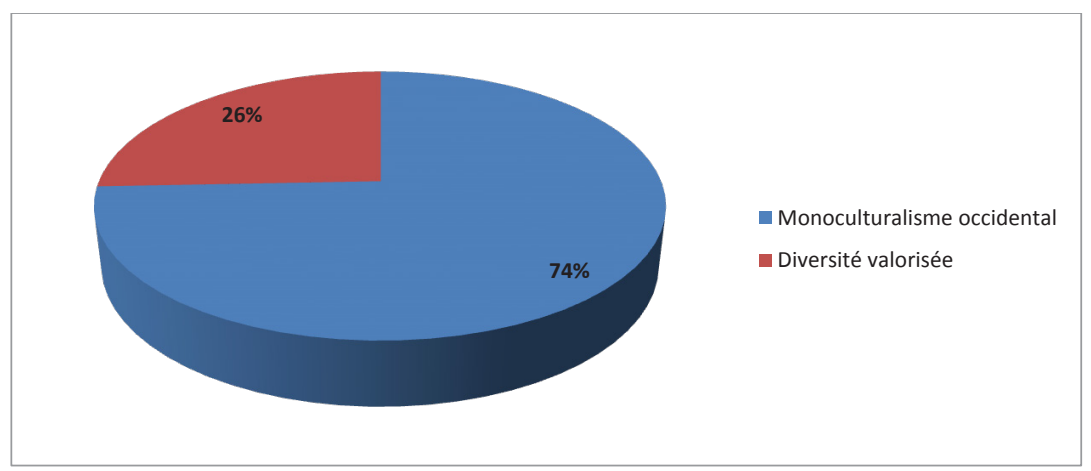

Image 3. Monoculturalisme et diversité véhiculés dans les publications. 
Sur les 446 publications de notre corpus, 160 ont un lien direct avec la mise en relief de référents culturels significatifs, isolés notamment à travers l'utilisation de référents facilement identifiables pour les internautes (comme les allusions à des séries anglo-saxonnes, par exemple, ou à des mangas japonais), ce qui fait un total de 35,87\% sur les prélèvements effectués. Cependant, sur ces 160 publications, 41 seulement valorisent une certaine forme de diversité culturelle, en tentant de jeter des ponts entre les référents susnommés ou en évitant de ne mentionner que des référents anglo-saxons ou purement occidentaux, alors que 119 montrent l'importance d'une sorte de monoculturalisme occidental, notamment dans les références constantes à certains films et à plusieurs séries télévisées. Si ces derniers sont évidemment diffusés dans plusieurs pays, ou au moins accessibles via internet (à travers des moyens plus ou moins légaux), ils deviennent des références aisément compréhensibles par un grand nombre d'utilisateurs ; en ce sens, les items culturels anglo-américains deviennent des vecteurs importants qui témoignent non seulement d'une faible valorisation de la diversité culturelle, tout en étant parfois porteurs (dans 1 cas sur 10, comme nous avons pu le souligner) de messages de discrimination. Il nous semble à ce titre opportun d'illustrer une nouvelle fois notre propos par des illustrations qui utilisent des références typiquement anglo-américaines (ou plus largement occidentales) pour la diffusion de messages discriminants :
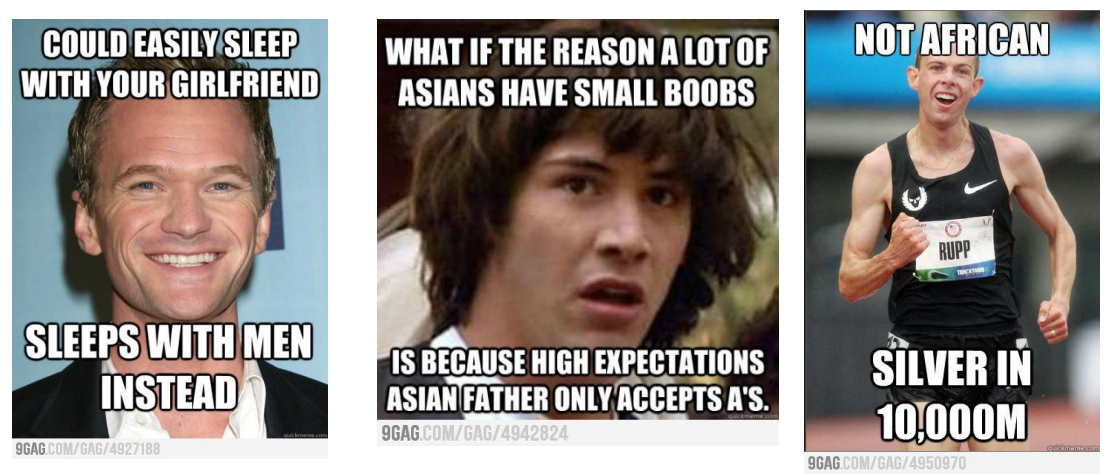

Illustration 2. trois exemples de publications discriminantes.

Ainsi, qu'il s'agisse de l'utilisation de l'image de l'acteur américain homosexuel Neil Patrick Harris (acteur de la série télévisée à succès «How I Met Your Mother ») pour promouvoir l'hétérosexualité, ou encore celle de Keanu Reeves pour une moquerie sur les seins des 
femmes asiatiques, en passant par les Jeux Olympiques comme vecteurs de stéréotypes raciaux, 9gag se retrouve dans une position largement polémique et finalement éloignée de l'idéal d'un internet permettant le rassemblement des peuples et la chute des barrières sociales ou culturelles.

\section{Conclusion : vers une négation de la diversité ?}

L'humour diffusé via 9gag permet, comme nous l'avons vu, de mettre en lumière et en image des messages qui montrent du doigt des catégories sociales, culturelles ou nationales distinctes :

- $\quad$ images de certains pays constamment dégradées (Roumanie et Philippines, notamment) ;

- $\quad$ image de la femme visiblement écornée : l'image de la femme véhiculée sur 9gag montre qu'elle devrait partager des loisirs comme les jeux vidéo, tout en obéissant à un stéréotype de femme active au foyer (avec les blagues récurrentes comme «bitch, make me a sandwich »);

- le dénigrement des questions de société liées à la communauté gay, avec notamment l'utilisation du terme " gay » comme dévalorisant et humiliant ;

- la publication de certaines réflexions humoristiques à caractère raciste ou xénophobe, même de manière indirecte.

A ce titre, la conclusion pourrait être la suivante : 9gag serait-il un site de mâle " geek » hétérosexuel blanc, ou au moins fortement occidentalisé ? Ce site populaire, qui se veut à la fois alternatif et rassembleur, montre qu'un outil de communication peut être utilisé à des fins différentes : si 9gag rassemble en effet, c'est par le biais d'images masculines et hétérosexuels, et aux dépens de certaines catégories ou minorités délibérément moquées. En ce sens, l'exemple de ce site internet montre que nous restons encore très éloignés de ce que Dervin appelle les «diverses diversités » et qui montre, en écho à la théorie des identités de Burke et Stets, que (Dervin, 2011, p. 112) les individus adaptent leurs rôles et leurs identités en jouant avec les contextes d'expression :

Il vaudrait mieux s'orienter vers l'étude de la négociation et de co-construction des diverses diversités des individus en présence 
plutôt que chercher des marques de la diversité 'culturelle', 'ethnique', 'linguistique' à travers des artefacts ou des discours de vérité (...). En résumé, travailler sur les processus plutôt que sur les produits.

En travaillant sur le site internet 9gag, nous avons travaillé non seulement sur des produits (à savoir des publications mises en ligne), mais également sur la manière dont certains processus ayant cours dans les sociétés sont négociées entre les individus ; ici, c'est notamment l'importance du vote des utilisateurs qui montre que la discrimination et le monoculturalisme restent des données partagées, voire encouragées parfois. Toutefois, notre étude des publications de 9gag laisse en suspens les questions que nous avions posées au début du présent article :

- il semble pratiquement impossible (ou assez difficile) de rassembler différents internautes sans utiliser des références culturelles communes, à moins que ces internautes n'aient pour but que de propager l'image d'une société androcentrée et hétérocentrée, qui se repose sur le partage de références anglo-américaines, suivant ainsi les pouvoirs économiques et financiers ;

- la grande diversité des utilisateurs de 9gag semble en revanche démontrer l'accessibilité quasi-universelle de certains référents anglo-américains, notamment à travers le partage de ces produits culturels par le biais du téléchargement internet, et l'adhésion des utilisateurs à un univers qui se retrouve de facto lié à une forme de modèle économique ;

- la combinaison des modèles STA et SPA, ainsi que l'utilisation du principe de traitement superficiel, permet effectivement de mettre en relief les liens sémantiques entre les référents utilisés par les internautes sur 9gag, et offre ainsi un approfondissement surprenant quant à la façon dont certaines idées sont partagées et popularisées sur le site.

Dans certaines publications, seuls les jeux vidéo et les mangas, d'origine japonaise pour leur écrasante majorité, sont parfois utilisés et partagés par une majorité d'utilisateurs de 9gag. Si ce site internet se veut transnational, il n'en reste pas moins à forte dominance occidentale, et semble propager une certaine forme de « culture occidentale», si tant est que ce concept puisse faire sens. 
En effet, l'étude de 9gag démontre également que les enjeux culturels masquent des enjeux de pouvoir importants (Sarangi, 1994), dont sont la cible les groupes sociaux les plus vulnérables, à savoir par exemple les femmes et les homosexuels. Ce point de vue peut également être proposé en abandonnant le concept de culture comme variable explicative des comportements sociaux (Wagener, 2012) :

The study of interactions and human behaviours and values is the study of lifeforms in action, which means that any satisfactory explanation should not depend on simplified categorizations. (...) In order to describe and study these expressions, the very concept of culture may even not be necessary at all ${ }^{1}$.

En effet, en évitant les filtres culturels, nous pouvons cerner les véritables enjeux de pouvoir à l'œuvre au sein des vecteurs de communication et de leurs messages. Le site communautaire 9gag n'échappe selon nous pas à cette règle, et invite à une vigilance concernant les souhaits des internautes regroupés à travers des forums alternatifs, qui peuvent constituer des sources d'expression libérées pour toutes formes possibles de discriminations ; cette vigilance est renforcée par le fait que les internautes semblent effectivement utiliser des référents qui favorisent le traitement superficiel de l'information et la validation de prédiscours, afin de consolider des nuages topiques qui permettent l'identification des internautes à l'univers de 9gag. En conclusion, si Internet permet le brouillage de certaines frontières sociales et géographiques, tout en modifiant profondément les moyens de communication des individus et groupes sociaux, la gestion parfois brutale de la diversité qui en résulte nous invite à penser l'émergence nécessaire de ce que Lemieux appelle une grammaire des sciences sociales (Lemieux, 2009), afin de comprendre les interactions en dehors de catégories parfois trop simplificatrices, et dont les individus s'emparent eux-mêmes afin de perpétuer certaines traditions discriminantes. Un tel projet, bien qu'ambitieux, permettrait d'éviter certains pièges décrits notamment par Raymond Boudon, comme le culturalisme, qui « inspire bien des politiques néfastes ; il obscurcit la connaissance de l'homme et des sociétés »(Boudon, 1995, p. 454).

1 «L'étude de interactions et des comportements et valeurs humains est en fait l'étude de formes de vie en action : cela signifie que toute forme d'explication satisfaisante ne devrait pas dépendre de catégorisations simplifiées. (...) Pour décrire et étudier ces manifestations, le concept de culture lui-même ne semble pas du tout être nécessaire $»$; notre traduction. 


\section{Références}

Angouri, J., \& Glynos, J. (2009). Managing Cultural Difference and Struggle in the Context of Multinational Corporate Workplace: Solution or Symptom? Working Papers in Ideology and Discourse Analysis, 26.

Boltanski, L., \& Thevenot, L. (1991). De la justification. Les économies de la grandeur. Paris : Gallimard.

Boudon, R. (1995). Le juste et le vrai. Paris : Fayard.

Bucholz, M., \& Hall, K. (2005). Identity and Interaction: a Sociocultural Linguistic Approach. Discourse Studies, 7(4-5), 585-614.

Burke, P. J., \& Stets, J. E. (2009). Identity Theory. Oxford : Oxford University Press.

Cap, P. (2010). Legitimisation in Political Discourse. Newcastle upon Tyne : Cambridge Scholars Publishing.

Conein, B. (1998), Les sens sociaux : coordination de l'attention et interaction sociale, Intellectica, 1/2(26/27), 181-202.

Dennett, D. (1990). Memes and the Exploitation of Imagination. The Journal of Aesthetics and Art Criticism, 48(2), 127-135.

Dervin, F. (2011). Impostures interculturelles. Paris : L'Harmattan.

Enfield, N. J., \& Levinson, S. C. (2006). Roots of Human Sociality. New York : Berg.

Frijda, N.H. (2003). Passions : l'émotion comme motivation. Dans J.-M. Colletta \& A. Tcherkassof (Ed.), Les émotions. Cognition, langage et développement, (pp. 15-32). Sprimont : Mardaga.

Galatanu, O. (2003). La sémantique des possibles argumentatifs et ses enjeux pour l'analyse du discours. Dans I. I. Las Heras \& M. J. Salinero Cascante (Ed.), El texto como encrucijada: estudios franceses y francofonos II, (pp. 213-226). Logrono: Publicaciones Universidad de La Rioja.

Kay, P. (1999). Intra-Speaker Relativity. Dans J. J. Gumperz \& S. C. Levinson (Ed.), Rethinking Linguistic Relativity, (pp. 97-114). Cambridge : Cambridge University Press.

Lemieux, C. (2009). Le devoir et la grâce. Paris : Economica.

Maillat, D., \& Oswald, S. (2009). Defining Manipulative Discourses: The Pragmatics of Cognitive Illusions. International Review of Pragmatics, 1(2), 348-370.

Meunier, J.-P. (2003). Approches systémiques de la communication. Bruxelles : De Boeck.

Mühlmann, H. (2005). Maximal Stress Cooperation. The Driving Force of Cultures. Vienna : Springer.

Paveau, M.-A. (2006). Les prédiscours: sens, mémoire, cognition. Paris : Presses Sorbonne Nouvelle.

Sarangi, S. (1994). Intercultural or Not? Beyond Celebration of Cultural Differences in Miscommunication Analysis. Pragmatics, 4(3), 409-427.

Wagener, A. (2012). Deconstructing Culture: Towards an Interactional Triad. Journal of Intercultural Communication, 29. 